\title{
ESTADO DEL ARTE - DESARROLLO DE UNA APLICACIÓN MÓVIL BASADA EN SISTEMAS DE REALIDAD AUMENTADA PARA LA VALIDACIÓN DE SISTEMAS DE INFORMACIÓN GEOGRÁFICA A NIVEL CATASTRAL
}

\section{STATE OF ART - A MOBILE APPLICATION DEVELOP- MENT BASED ON SYSTEMS OF AUGMENTED REALITY FOR THE VALIDATION OF GEOGRAPHIC INFORMATION SYSTEMS ON CATASTRAL LEVEL}

\section{RESUMEN}

La realidad aumentada en dispositivos móviles es un concepto que a través de los años ha ido tomando un valor significativo cada vez más alto, esto sumado al desarrollo de los sistemas de información geográfica y el catastro, ha llevado a un crecimiento de las mismas pasando de salas exclusivas de investigación, a ser parte del uso común, y dentro de poco, al uso específico en distintas áreas del conocimiento científico. El presente artículo presenta el estado del arte para el desarrollo de una aplicación móvil basada en sistemas de realidad aumentada para la validación de sistemas de información geográfica a nivel catastral, se logró encontrar bastante información sobre el tema que da puntos de partida para el desarrollo de esta temática, la cual está en un punto importante para encontrar una nueva solución para el manejo de la información geográfica a nivel catastral.

Palabras clave: Realidad Aumentada, Sistemas de Información Geográfica, Catastro, Aplicación móvil Android.

\begin{abstract}
The augmented reality on mobile devices is a concept that through the years has been taking an increasingly high significant value, this added to the development of geographic information systems and land, has led to a growth of the same going from exclusive research rooms, become part of common usage, and soon, the specific use in different areas of scientific knowledge. This paper presents the state of the art for the development of a mobile application based on augmented reality systems for validation of GIS to cadastral level, we were able to find enough information about the subject that gives points for the development of this subject, which is an important point to find a new solution for managing geographic information cadastral level.
\end{abstract}

Keywords: Augmented Reality, GIS, Cadastre, Android Mobile Application.

\section{Sergio Ramirez Navarro:}

Ingeniero Catastral y geodesta - Universidad Distrital Francisco José de Caldas - Colombia. Estudiante Maestría en ciencias de información y las comunicaciones- Universidad Distrital Francisco José de Caldas - Colombia,

Profesor- Universidad Distrital Francisco José de Caldas - Colombia - sergio.ramirez@incige.com

Tipo: Revisión de tema

Fecha de Recepción: : 4 de Abril de 2015 Fecha de Aceptación: : 26 de Junio de 2015 


\section{INTRODUCCIÓN}

Los sistemas basados en realidad aumentada han ido avanzando cada vez más a través de los años, de la misma forma los Sistemas de información geográfica en catastro, lo cual ha llegado a un punto donde una combinación de estas áreas posibilitará el desarrollo de una solución que permita validar datos de un sistema de información geográfica, a través de una aplicación móvil de realidad aumentada. Hoy en día se ha venido trabajando en estas dos temáticas, pero el tema del catastro ha sido poco tocado, el avance de estos temas ha logrado que se llegue a un punto donde se puede crear una nueva aplicación que logre suplir este vacío del conocimiento en el manejo de la información catastral, la cual en muchos casos puede llegar a ser difícil y engorrosa de validar. Al tener una aplicación móvil desarrollada en este sentido se podría llegar a una solución innovadora que mejore el entendimiento en estas áreas y también el trabajo profesional en las mismas. El objetivo de este artículo es presentar el estado del arte del desarrollo de esta temática, dar a conocer la importancia de la misma y de lo que significa la creación de esta aplicación a futuro.

\section{DESARROLLO}

\section{A. Antecedentes}

La realidad aumentada (Augmented Reality, $\mathrm{AR}$ ) permite a los usuarios interactuar con el mundo real y con objetos generados por computador lo cuales son visualizados en entornos tridimensionales [1] [2]. Este es un concepto que viene trabajándose desde hace ya varios años, que con el paso del tiempo, del desarrollo constante de la tecnología, de hardware y software, ha ido mejorando cada vez más. La realidad aumentada permite modificar el mundo como se percibe de forma individual gracias a la agregación de alguna información digital o almacenada en Sistemas de información [3].

Los sistemas de información (SI) pueden ser manejados desde un punto de vista funcional como una serie organizada de elementos que proveen información, para luego ser agrupa- dos, clasificados, procesados y transmitidos. Estos sistemas han sido desarrollados para manejar tantos niveles de complejidad como se requiera [3]. Los sistemas de información pueden ser convertidos en Sistemas de información geográfica (SIG), ya que básicamente los SIG son sistemas de información que pueden organizar y presentar información alfanumérica espacialmente referenciada [4]. Basados en distintas bases de datos, estos sistemas proporcionan información geográfica digital a ser adquirida digitalmente. Ello permite el análisis de ésta por manipulación e interrogación [3].

Al combinar los sistemas de realidad aumentada con los sistemas de información geográfica se obtienen potenciales de estudio bastante elevados, ya que se permite la visualización del entorno de manera más aplicativa, permitiendo entender la realidad de una manera distinta, modificando la forma como los SIG han venido siendo utilizados. Usualmente en su visualización, los SIG requieren básicamente de dos elementos de entrada [3]:

- $\quad$ Petición del usuario.

- $\quad$ Parámetros de despliegue (factores de escala, abstracción, simbolizaciones, etc.).

Para el despliegue de los SIG usando AR, se debe agregar un nuevo componente que debe ser tenido en cuenta [3]:

\section{- $\quad$ La posición del usuario.}

Para obtener información desde un SIG, el usuario no solamente debe lanzar una petición correcta, debe también dar una posición y orientación correcta. Esto le da a los SIG un amplio campo de acción en los sistemas de AR [3]. Existen dos entornos de trabajo para los cuales se podría combinar la AR con los SIG:

1. Los entornos cerrados de información geográfica, básicamente pueden relacionarse con las tecnologías de mapas aumentados, los cuales permiten ver más allá de un simple mapa análogo (mapa en papel), ya que ahora se puede visualizar cartografías en 3 dimensiones de forma digital en un entor- 
no real. Estos en su mayoría se han venido desarrollando a través de marcadores que permiten guiar la información digital con la que se presenta en la realidad [5] [6].

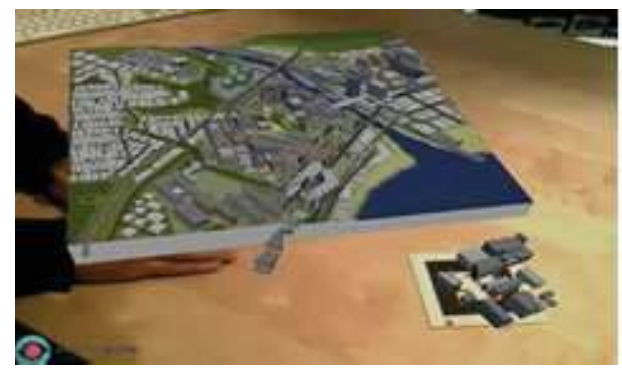

Figura 1. Realidad Aumentada en Entornos Abiertos [7]

Fuente: J. C. Sierra R., «http://juanchosierrar. blogspot.com/,» 01 Abril 2014. [En línea]. Available: http://juanchosierrar.blogspot. com/2013/02/liberacion-de-la-api-armediapara-crear.html.

2. Los entornos abiertos de información geográfica [8], se refieren directamente a la interacción con la realidad del entorno (construcciones, vías, relieve o elementos cartografiables), en este sentido se han realizado gran cantidad de proyectos mostrando las capacidades de combinar los sistemas de RA con los SIG, un ejemplo de como el de [9] donde se propone mostrar un sistema de RA con visión indirecta de información geográfica incrustada (nombres de vías y números, nombres de monumentos, etc.). En [10] se muestra el diseño de una aplicación que permite mostrar de forma de etiqueta la altura de picos de montañas que se presentan en el entorno del sistema de RA. Asimismo existen proyectos de visualización de infraestructuras (tuberías, edificaciones, redes, etc.), que muchas veces no pueden ser observadas con claridad, bien sea porque no son visibles directamente por el ojo humano, o también porque no se conocen sus características dimensionales [11] [12] [13] [14], esto se puede apreciar en la siguiente Imagen.

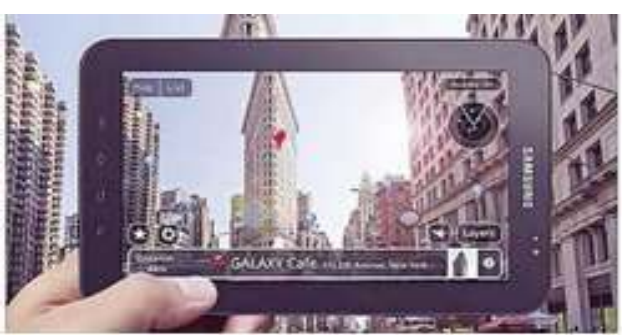

Figura 2. Sistmas en tiempo real Fuente: ecodiario.eleconomista.es/, «http:// ecodiario.eleconomista.es/,» 01 Abril 2014. [En línea]. Available: http://ecodiario.eleconomista.es/tecnologia/noticias/5586973/03/14/ Las-animaciones-en-3D--saltaN-a-la-publicidad.html\#.Kku8fT6m98Xmvxc.

Además de esto, se ha venido trabajando en sistemas de RA con SIG utilizando conexión de móviles a servidores web con lo cual se puede hacer una validación de datos de gran tamaño sin llenar en muchos casos las capacidades de estos dispositivos que a veces tienden a ser limitados en su capacidad de almacenamiento.

En Colombia, [16] desarrolló una aplicación móvil para el celular de la marca Apple Inc. IPhone 3g., donde se agrega información urbana a la visión común del usuario, esta información corresponde a datos geográficos de las edificaciones debidamente legalizadas ante las entidades gubernamentales, esto a través de conexiones a un servidor de información 3D que contiene la base de datos espacial. Con esta información se valida si se encuentra actualizada o existen posibles construcciones ilegales, lo cual puede ser de gran ayuda en estudios catastrales.

Dicha aplicación se desarrolló pero se encontró un elemento el cual quedó señalados como trabajo futuro por mejorar, éste es la desalineación de los objetos en 3D. Esto se debe a que los sensores del dispositivo móvil entregaban la información con cierto retraso y error en la medición. Mediante el uso de esquemas de procesamiento de imágenes para detectar los bordes de los edificios, y una identificación de a cuál edificio pertenecen, se podría lograr una mejor alineación [16]. Para esto se podrían implementar los métodos de [17] [18], asimis- 
mo se podría trabajar en el Sistema operativo de móviles Android, el cual tiene menos limitaciones en cuanto al uso de las herramientas del dispositivo móvil [19]. Lo que se pretende es desarrollar una aplicación móvil basada en Sistemas de realidad aumentada que permita la validación de sistemas de información geográfica a nivel catastral en el sistema operativo Android, mejorando la aplicación desarrollada por [16] la cual en lo hablado por su autor, debía alinear los elementos en 3D de forma manual y no automática, al realizarse esto se podría verificar de forma más precisa la información catastral.

\section{B. Conceptos}

Para entrar en el desarrollo de la revisión del estado del arte, se requiere conocer algunos conceptos fundamentales. Los cuales servirán como base para el desarrollo de la investigación.

\section{1) SIG}

Un Sistema de Información Geográfica o SIG por sus siglas es definido en Olaya (2011) como un sistema de información diseñado para trabajar con datos referenciados mediante coordenadas espaciales o geográficas.

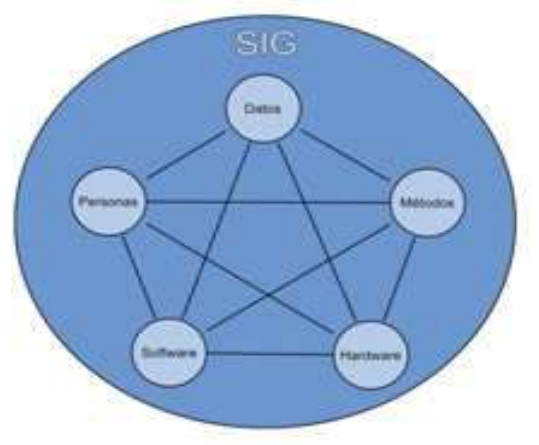

Figura 3. Elementos que conforman un Sistema de información geográfica [20].

Fuente: V. Olaya, Sistemas de Información Geográfica, OSGeo, 2011.

En otras palabras, un SIG es tanto un sistema de base de datos con capacidades específicas para datos georreferenciados, como un conjunto de operaciones para trabajar con esos datos. En cierto modo, un SIG es un mapa de orden superior.

\section{2) Catastro}

Catastro es definido por el Instituto Geográfico Agustin Codazzi (2011) como el inventario o censo, debidamente actualizado y clasificado, de los bienes inmuebles pertenecientes al Estado y a los particulares, con el objeto de lograr su correcta físcica, jurídica, fiscal y económica. Desde el aspecto físico consiste en la identificación, descripción y clasificación del terreno y las edificaciones del predio sobre documentos gráficos, tales como cartas, planos, mapas, fotografías aéreas, ortofotografías, espaciomapas, imágenes de radar o satélite u otro producto que cumpla con la misma función [21].

Desde el aspecto jurídico consiste en indicar y anotar en los documentos catastrales la relación entre el sujeto activo del derecho, o sea el propietario o poseedor, y el objeto o bien inmueble, mediante la identificación ciudadana o tributaria del propietario o poseedor, y de la escritura y registro o matrícula inmobiliaria del predio respectivo. [21]

Desde el aspecto económico consiste en la determinación del avalúo catastral del predio, obtenido por la adición de los avalúos parciales practicados independientemente para los terrenos y para las edificaciones en él comprendidos [21].

Desde el aspecto fiscal consiste en la preparación y entrega a los Tesoreros Municipales o quien haga sus veces y a las Administraciones de Impuestos Nacionales respectivas, de los listados de los avalúos sobre los cuales ha de aplicarse la tasa correspondiente al impuesto predial unificado y demás gravámenes que tengan como base el avalúo catastral, de conformidad con las disposiciones legales vigentes.

\section{3) Realidad Aumentada}

Realidad Aumentada o RA por sus siglas es definido en [2] como un medio en el que una 
información es agregada al mundo físico en registro con el mundo, el cual requiere tres características esenciales para definirla:
a. Combinar lo real y lo virtual
b. Interactuar en tiempo real
c. Registrado todo en 3 dimensiones

En [3] se define la Realidad aumentada como visualización directa o indirecta en tiempo real del entorno del mundo físico que ha sido realzado por agregación de información virtual generada de forma computarizada a este.

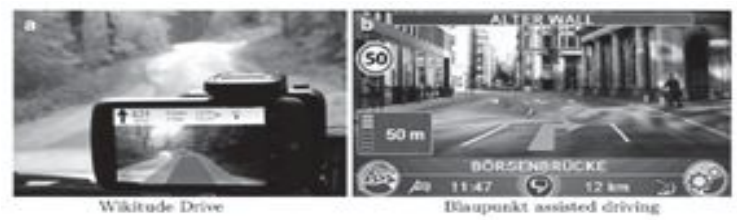

Figura 4. Aplicaciones de asistencia de manejo de carros combinando SIG y AR [3].

Fuente: B. Furhn, Handbook of Augmented Reality, Boca Ratón, Florida: Springer, 2011.

4) Sistema operativo Android Android es un sistema operativo móvil basado en Linux, que junto con aplicaciones middleware está enfocado para ser utilizado en dispositivos móviles como teléfonos inteligentes, tabletas, Google TV y otros dispositivos. Es desarrollado por la Open Handset Alliance, la cual es liderada por Google [22].

\section{REVISIÓN DEL ESTADO DEL ARTE}

El avance en la forma como se visualiza la información geográfica utilizando bien sea por dispositivos móviles, por realidad aumentada o por computador ha ido mejorando con el paso del tiempo, y con esto el desarrollo de nuevas tecnologías que han llevado a la resolución de problemas y de la formulación de nuevos. Recientemente en la parte de SIG se encuentran investigaciones como la de Min, Mei, Feizhou, Zhipeng, \& Daozheng (2007), la cual implementaba modelos detallados y mapas en 3D del mundo real, por medio de un programa llamado ARGIS, el cual utilizaba datos de receptor GPS y sensores incerciales, posición del observador y la orientación, de esta forma registrando elementos en 3D [23]. En este mismo año Sadoun \& Al-Hanbali (2007) realizaron una modelación de SIG para edificios altos y sus aplicaciones, gracias a estos modelos lo que buscaron los autores era demostrar la eficacia en el modelado 3D para catastro con fines de evaluación de bienes raices, al final el proyecto se limitó debido al software y a hardware, pero presento resultados aceptables para muchas aplicaciones en modela de la ciudad, tales como las aplicaciones de catastro [24].

En el año 2008 se realizaron proyectos relacionados con realidad aumentada y manejo de aplicaciones móviles, uno de ellos fue el realizado por $\mathrm{Wu}, \mathrm{Du}, \&$ Ren (2008) el cual fue uno de los primeros trabajos realizados sobre realidad aumentada e información geoespacial, a través de librerías como el ARtoolkit se pudo implementar la visualización de RA de datos geoespaciales, abriendo posibilidades a muchas áreas no solo del conocimiento espacial sino también del uso de universidades, laboratorios, museos, comunidad artística o inclusive, de los militares (Wu, Du, \& Ren, 2008). Tambien se encuentra el proyecto de $\mathrm{Li}$, Yang, \& $\mathrm{He}$ (2008) donde se empezó a trabajar en una investigación sobre aplicaciones de bases de datos móbiles basadas en SIG, donde se encuentran elementos sobre la conexión de los datos, la transmisión e interacción y la sincronización de la base de datos móvil, encontrando como limitación las velocidades de las redes que se tenían para ese momento [25].

En el año 2009 se encontró que proyectos como el de Bhattacharyya, Chakraborty, Bandyopadhyay, \& Kim (2009) donde se trabajó en consultas móviles para Sistemas de Información Geográfica, en esta investigación se desarrolló un algoritmo de búsqueda de la situación de un determinado punto de coordenadas con respecto a una superficie. También se trabajó en una investigación basada en la colección de información sobre plataformas móviles de SIG, en este trabajo se pudo hacer recolección de información para SIG con herramientas móviles como un PDA, donde se pudo almacenar información espacial y se representó visualmente 
dentro de la plataforma, dándole una nueva oportunidad para el desarrollo de servicios móviles de información espacial [26].

En el 2010 se siguió investigando sobre todo lo que son las características del SIG móvil, donde se encontró que los avances que vienen realizándose en términos de telecomunicaciones le han dado un enfoque mucho más fuerte a este tipo de aplicaciones, rompiendo paradigmas en el conocimiento a tal punto de afectar la noción de la vida humana, ya sea en el entretenimiento, el transporte inteligente, los viajes y demás, asimismo su importancia dentro del campo de acción geoespacial [27].

En el año 2011, un proyecto desarrollado dentro de este enfoque móvil fue el de una integración de una base de datos SIG catastral en un sistema de navegación GPS para localizar terrenos en levantamientos catastrales obteniendo información para facilitar las visitas a estos terrenos y permitiendo realizar actualizaciones, algo en lo que se venía trabajando en años anteriores, pero que no se había podido hacer en trabajos reales, beneficiando a inspectores de catastro y también a otros usuarios [28]. También en este año se logró el desarrollo del estado del arte de los Sistemas de información geográfica y la realidad aumentada, donde se encontraron las grandes capacidades que tienen estas dos tecnologías juntas, y en las que además se argumentó sobre muchos de los elementos que aún quedan por mejorar como por ejemplo el trabajo con dispositivos móviles, la alineación correcta de los objetos y el retraso en la sincronización de la información virtual sobre la real [3].

Ya para el año 2013 se empiezan a ver resultados de trabajos de realidad aumentada sobre Sistemas de información geográfica, un caso de estudio fue el de registro y seguimiento de la composición de especies de árboles, en este proyecto propone una aplicación de seguimiento de móviles (artsc) que integra la información geográfica y técnica móvil de realidad aumentada para el seguimiento de la composición de especies de árboles. Mediante el uso de la cámara en el teléfono inteligente, la información sobre la composición de las especies se superpone en la parte superior de la imagen real. Por lo tanto, esta aplicación puede ser uno de los métodos alternativos a los investigadores a seguir la información sobre las especies de árboles. Esta aplicación representa un gran avance en el tema del manejo de los sistemas de RA y SIG, haciendo más sencillo e intuitivo el trabajo de seguimiento de especies de árboles, reduciendo los tiempos de trabajo y representando beneficios para la enseñanza superior a futuro [29].

\section{5) Realidad aumentada}

En realidad aumentada se viene trabajando desde hace bastante tiempo, pero estas labores de investigación se han venido desarrollando en laboratorios o para uso de unos pocos, llegando a resultados poco vistosos hasta la llegada del nuevo milenio, en los últimos 5 años el desarrollo de esta tecnología ha crecido de una manera que antes no se habría podido pensar, teniendo en cuenta estos datos se hablará de los avances que se han ido realizando a través de los años en esta área.

De los años 90 a 2005 se ha venido trabajando en las primeras formas de trabajo de Realidad aumentada con Sistemas de información geográfica, en Chen \& Shibasaki (1999) se marcaron las pautas para el desarrollo de esta tecnología donde se habló de los sensores que debe tener el sistema para funcionar de forma eficiente, estos son una cámara, un sistemas de posicionamiento global (GPS), un sistema de navegación inercial (INS), magnetómetro, giroscopio y tecnología de navegación de imagen. Los resultados fueron bastante importantes para ese momento, pero el limitante de hardware tecnológico imposibilitó un mejor desarrollo por varios años [30]. Unos años más adelante, con el desarrollo de los primeros teléfonos móviles con cámara digital, se logró hacer las primeras pruebas de software de realidad aumentada sobre estos mismos, los resultados de esta investigación mostraron que aún se requería de mejor hardware para obtener datos confiables y de mejor calidad [31]. En el 2005 se desarrolló una aplicación que permitía ver información espacial para el control de viñe- 
dos, todo esto a través de sistemas de realidad aumentada, la cual permitía ver datos en 3D al aire libre utilizando un equipo con trípode. En este caso no se usaron dispositivos móviles sino aparatos un poco más grandes, esto con el fin de evitar las limitantes de hardware que tenían éstos para ese año [32].

Pasaron varios años para el desarrollo de mejores tecnologías para la implementación de aplicaciones de realidad aumentada, esto teniendo en cuenta las limitantes de hardware que existían, que imposibilitaban una movilidad adecuada que hiciera el trabajo mucho más sencillo de las personas que empleaban éstas. Hasta el año 2010 se encontraron resultados nuevos sobre mecanismos y mentodos para la visualización y representación de información espacial en entornos abiertos con realidad aumentada, donde lo que se buscó fue aplicar los elementos y sensores descritos en años anteriores, los cuales ya podían ser implementados teniendo en cuenta que existe un hardware mucho más avanzado que años anteriores, dando pie para convertir a la realidad aumentada en entornos abiertos como una nueva interfaz para la representación espacial para la visualización de la información (Wu \& Ren, Mechanism and Methods of Outdoor AR Spatial Information Visualization Representation, 2010).

En el año 2011 se presenta el gran auge de esta tecnología donde se encuentran grandes avances no sólo a nivel de hardware sino también de software, uno de ellos trabaja realidad aumentada en escenas urbanas en 3 dimensiones, el cual es el primer sistema completamente funcional, este aplica bases de datos escalables, modelos 3D y metadatos, el sistema sólo presenta un poco de retrasolo cual generaba que el emparejamiento de la información a veces tardara un poco más, lo cual según los autores quedó para trabajo futuro [33]. También se desarrollaron aplicaciones que permitían hacer seguimiento de elementos 3D en realidad aumentada por medio de dispositivos móviles, este fue un trabajo esperimental con lo cual se obtuvieron resultados iniciales con elementos pequeños, a futuro los autores pretenden mejorar su robustez utilizzando otras señales visua- les, así como ampliar el trabajo que se presenta con el fin de apoyar el modelado In situ y de creación para aplicaciones interactivas de realidad aumentada.

Otro de los elementos importantes que se dio en el 2011 fue la implementación de un framework (marco de trabajo para desarrollo) para realidad aumentada en dispositivos móviles, donde se abrio paso al código abierto para el desarrollo de bibliotecas basadas en seguimiento para realidad aumentada, donde se propone un algoritmo OpenCV(desarrollado para realidad aumentada) y OpenGL(desarrollado anteriormente para el manejo de gráficos en 3 dimensiones) el cual puede ser implementado en plataformas Android, IOS (Apple Inc.) o Windows Mobile. Este es un desarrollo inicial por lo que no significa que aún no exista trabajo futuro, los desarrolladores de este algoritmo buscarán implementar a futuro un componente de seguimiento de la historia en el algoritmo para refinar la velocidad del sistema, así como también el proporcionar el seguimiento de objetos reales para la aplicación correcta de la realidad aumentada en dispositivos móviles [34]. También se encontró la implementación del procesamiento de imágenes y realidad aumentada para teléfonos inteligentes. En este desarrollo se implementó la librería OpenCV así como otras librerías como son ARtoolkitPlus y VRtoolkit, este desarrollo sirve como base para la detección de rostros, reconocimiento de marcadores y avances en software de realidad aumentada [35].

Un proyecto que también se desarrolló este año fue el de reconocimiento escalable en tiempo real y seguimiento basado en un modelo servidor-cliente para realidad aumentada móvil, en este proyecto se encontró que en muchos casos el almacenamiento de los dispositivos móviles es una limitante en el manejo de grandes volúmenes de información, y a su vez el continuo desarrollo de las telecomunicaciones hoy permite establecer conexiones con servidores remotos que puedan almacenar y escalar esta información, haciendo más vérsatil el uso de los dispositivos móviles, algo realmente importante para el manejo de sistemas de reali- 
dad aumentada, donde se lograron establecer conexiones y se logró manejar la información de forma escalable de tal manera que fuera más facil de manejar para el dispositivo móvil [36].

En este mismo año se desarrolló el proyecto de Wilches (2011), el cual es la base para el desarrollo de la presente investigación, se puede encontrar que este proyecto maneja varios de los elementos de los proyectos que se vinieron desarrollando durante este año, y también sobre las limitaciones que tuvo este mismo para el seguimiento de objetos en 3D en tiempo real, así como el retardo en la sincronización de los datos [16].

En el año 2012 también se trabajo bastante en esta área, se desarrollaron aplicaciones de realidad aumentada para dispositivos Android orientados a la educación de niños de básica primaria [37]. Además se trabajó en un proyecto que hace un registro interactivo de realidad aumentada en sistemas de información geográfica, utilizando SIG 3D y Realidad aumentada aplicada a exteriores, este proyecto se realizó con sensores diferentes a dispositivos móviles, pero plantea la idea de manejar el software de forma más efectiva en estos mismos [38].

Otro proyecto desarrollado en este año consistio en un descriptor binario altamente eficiente llamado Local diferences Binary (LDB) el cual calcula directamente una cadena binaria de un parche de imagen usando intensidad simple y pruebas de diferencia de gradiente en las cuadrículas en pares dentro del parche. Este permite manejar de forma más rápida y eficiente los datos escalables de realidad aumentada en dispositivos móviles, para este caso utilizaron dispositivos como tablets android el cual mostró resultados bastante rápidos. Asimismo los autores proponen como trabjo futuro la exploración de un método más avanzado enla selección de bits basado en aprendizaje automático y la implementación de otros elementos como la invariancia de rotación, la cual permitiría reducir aún más los tiempos para la deteción y descripción de las funciones de consulta [39].

También se trabajó en proyectos basados en seguimiento sin marcadores para realidad aumentada en dispositivos móviles, esto quiere decir que se trabajó en una aplicación que permitiera la detección de objetos reales sin la necesidad de utilizar marcadores, los cuales son bastante utilizados en este tipo de tecnologías [40].

En este mismo año se trabajó en las primeras aplicaciones de realidad aumentada basadas en computación en la nube, esto facilita el procesamiento de la información, ya que es una máquina remota la que procesa la información y no el dispositivo móvil en sí, su limitación es la velocidad de las conexiones que permita un procesamiento eficiente de lo que se consulte, en este caso se hizo énfasis para entornos cerrados, pero se abrió la puerta a una nueva forma de desarrollo en esta área, que a futuro puede ser aplicada a entornos abiertos [41].

Por último se presentó un proyecto basado en seguimiento inercial para aplicaciones de realidad aumentada, en esta investigación se aprovecharon los sensores inerciales que tienen lo dispositivos móviles, así como el uso de las cámaras para mejorar el seguimiento y la captura de información para RA, el trabajo es inicial pero pretende unificar todos los sensores del dispositivo para mejorar aún más el seguimiento de los objetos en el mundo real [42].

Como se pudo observar en el desarrollo de esta revisión del estado del arte, se han realizado muchas investigaciones relacionadas con Realidad aumentada y SIG, así como también últimamente el uso de dispositivos móviles para la utilización de estas aplicaciones, pero se encuentra que aún no existe una solución al problema presentado por Wilches (2011), por lo cual aún existe un vacío en el conocimiento. Una mejora en el sentido que propone este proyecto, significa un avance en el desarrollo de aplicaciones de Realidad aumentada enfocadas a Sistemas de información geográfica a nivel catastral, en el siguiente capítulo se definirá el marco teórico que servirá de guía para el desarrollo de la investigación y de la forma como se realizará la aplicación que se propone para este proyecto. 


\section{CONCLUSIONES}

Al realizar la revisión del estado del arte, se ha podido encontrar que existe un vacío del conocimiento en aplicaciones móviles en sistemas basados en realidad aumentadas y sistemas de información geográfica a nivel catastral. Se ha venido trabajando en aplicaciones de este tipo, pero no existe una aplicación que permita validar la información geográfica con una exactitud significativa (existen aún inconvenientes con la alineación de objetos en 3D), es por esto que se haya la necesidad de un desarrollo que mejore las aplicaciones anteriores, brindando una solución que permita una validación efectiva de la información catastral a través de sistemas de realidad aumentada.

Teniendo en cuenta esto el trabajo futuro en esta área se encuentra principalmente en una aplicación sólida que permita la validación de datos geográficos, utilizando alineación de objetos en 3D, con conexiones eficientes a servidores escalables (a través de servicios Web), y que pueda ser trabajada más adelante por entidades estatales, no solo a nivel Colombia, sino a nivel internacional

\section{Referencias}

[1] L. Hassaine, "Implementación de un Control Digital de Potencia Activa y Reactiva para Inversores Aplicación a Sistemas Fotovoltaicos Conectados a Red", tesis doctoral, Universidad Carlos III de Madrid, Madrid, 2010.

[2] H.R. Murcia. "Desarrollo de la energía solar en Colombia y sus perspectivas". Revista de ingeniería. n.28. 2008. pp. 83.

[3] I. L. Kosow, "Máquinas eléctricas y transformadores", Segunda edición, Pearson educación, 1993.

[4] S.B. Kjaer, J.K. Pedersen, F. Blaabjerg, "A review of single-phase grid connected inverters for photovoltaic modules," Industry Applications, IEEE Transactions on, vol.41, no.5, pp. 1292- 1306, Sept.Oct. 2005.
[5] M. Calais, J. Myrzik, T. Spooner, V.G. Agelidis, "Inverters for single-phase grid connected photovoltaic systems-an overview," Power Electronics Specialists Conference, 2002. pesc 02. 2002 IEEE 33rd Annual, vol.4, pp. 1995-2000, 2002.

[6] F. Blaabjerg, R. Teodorescu, Z. Chen, M. Liserre, "Power converters and control of renewable energy systems," 1ra ed, pp. 1-120, 2004.

[7] D.E. Álvaro, "diseño de un inversor monofásico autónomo de baja frecuencia ajustable mediante bus DC", proyecto fin de carrera, Departamento de ingeniería eléctrica, universidad Carlos III de Madrid escuela politécnica superior, Madrid, 2009.

[8] S.Y.R.Hui, Senior Member, IEEE, I. Oppermann, and S. Sathiakumar, "Microprocessor-Based Random PWM Schemes for DC-AC Power Conversion," ieee transactions on power electronics, vol. 12, no. 2, pp. 253-260, march 1997.

[9] Y.Xue, L.Chang,S.Baekhoej, J.Bordonau, T.Shimizu, "Topologies of Single-Phase Inverters for Small Distributed Power Generators:An Overview". Power Electronics, IEEE Trasactions, vol.19, pp.1305-1314, September 2004.

[10] C.L. Trujillo, " Concepción de controladores conFigurables para microinversores fotovoltaicos operando como unidades autónomas de generación de energía en microrredes," tesis doctoral, Departamento de ingeniería electrónica, universidad politécnica, Valencia, 2011

[11] J.M.A. Myrzik, M.Calais, "String and Module Integrated Inverters for Single-Phase Grid Connected Power Tech Conference, 2003 IEEE Bologna, vol. 2, pp 8, June 23-26, 2003

[12] S.M. Silva, B.M. Lopes, B.J. Filho, R.P. Campana and W.C. Bosventura, "Performance evaluation of PLL algorithms for single-phase 2004. 39 th IAS annual meeting. Conference record of the 2004 IEEE, vol 4, pp 2259-2263, 3-7, Oct 2004. 